\title{
MOLUSCOS EM CONTEXTOS ARQUEOLÓGICOS PORTUGUESES: IMPORTÂNCIA E ESTADO DA ARTE
}

\author{
PEDRO M. CALLAPEZ ${ }^{(1)(2)}$, RICARDO PIMENTEL ${ }^{(3)} \&$ PEDRO A. DINIS $^{(1)(4)}$
}

\begin{abstract}
Resumo:
O estudo integrado conchas de moluscos presentes em sítios arqueológicos constitui um dos principais aspetos da Zooarqueologia. A abordagem metodológica dos contextos de campo e das amostragens recolhidas compreende vários tópicos interligados, incluindo a sistemática e taxonomia, a tafonomia, a análise estatística e biométrica, a paleoecologia de indivíduos, populações e paleocomunidades e a análise paleobiogeográfica e paleoclimática, para além de outros relacionados com a intervenção humana, incluindo o consumo e a economia alimentares, e a utilização como utensílios e adornos. No decurso das últimas duas décadas, estes aspetos têm vindo a ser progressivamente implementados no estudo interdisciplinar de contextos arqueológicos portugueses de diferentes idades, revelando a importância emergente das análises integradas e transversais, fruto de uma colaboração mais ativa entre os arqueólogos e paleontólogos e biólogos interessados em faunas do Plistocénico e Holocénico.
\end{abstract}

Palavras-chave: Mollusca, Bivalvia, Gastropoda, Zooarqueologia, Portugal.

Abstract:

\begin{abstract}
Molluscs in Portuguese archaeological contexts: importance and state-of-the-art
The integrated study of molluscan shells found in archaeological sites is one of the main aspects of Zooarchaeology. The methodological approaches used for field contexts and collected bulk samples consist of several related topics, including systematics and taxonomy, taphonomy, statistical and biometric analysis, palaeoecology of individuals populations and palaeocommunities, and palaeobiogeographic and palaeoclimatic analysis, besides other topics related with human intervention, including food consumption and economy, and tool and adornment usage. During the last two decades, these aspects have been gradually implemented as part of interdisciplinary studies dedicated to Portuguese archaeological contexts of different ages. This fact reveals the emergent importance of integrated and transversal analyses, as a result of a better collaboration between the archaeologists, and palaeontologists and biologists interested in Pleistocene and Holocene faunas.
\end{abstract}

Keywords: Mollusca, Bivalvia, Gastropoda, Zooarchaeology, Portugal.

Received: 21 March, 2016; Accepted: 26 April, 2016

\section{INTRODUÇÃO}

Os moluscos acompanham as vivências quotidianas do Homem desde o alvor da Pré-História, numa relação que se enraizou em hábitos, gostos e tradições milenares, parte das quais chegaram até aos nossos dias. Graças à sua grande abundância e facilidade de captura através de práticas rudimentares de recoleção ou de exploração de viveiros, desde sempre constituíram um recurso estratégico, capaz de suprir eficazmente a dieta de grupos ou comunidades de indivíduos, como complemento à pesca e à caça, ou ainda a atividades de produção de alimentos ligadas à agricultura ou à pecuária.

Não se estranha, por conseguinte, que as conchas de moluscos marinhos, com destaque para os das classes Bivalvia e Gastropoda, integrem os espólios paleozoológicos de muitos contextos arqueológicos portugueses, contribuindo para a sua melhor interpretação e reconstituição. Assim como em Espanha (GARCIA et al. 2010), o historial desta concomitância e dos estudos dela derivados remonta à segunda metade do século XIX, aquando da descoberta e exploração científica dos primeiros concheiros no vale do Baixo Tejo, por parte de Francisco António Pereira da Costa (1809-1888), de Carlos Ribeiro (1813-1882) e de outros pioneiros da Arqueologia portuguesa que se lhes seguiram (e.g. COSTA 1865; RIBEIRO 1884; OLIVEIRA 1892; CORRÊA 1931; PAÇO 1938; BREUIL \& ZBYSZEWSKI 1947; ROCHE 1951, 1953a, 1953b, 1960 , 1972; FERREIRA 1956).

Também as conchas de moluscos terrestres e de água doce, sobretudo dos primeiros, são presença frequente em sítios arqueológicos. Nesse sentido, a sua existência e significado têm vindo a ser focados desde os primeiros estudos arqueológicos portugueses, como o demonstram, por exemplo, as observações de Joaquim Filipe Nery Delgado (1835-1908) sobre conchas de "Helix nemoralis" presentes na necrópole neolítica da gruta de Casa da Moura (Delgado 1867).

Estes primeiros estudos e todo um manancial bibliográfico que se tem multiplicado, em Portugal e a nível internacional, sobretudo nas últimas décadas, demonstram que os moluscos são um dos prin-

(1) Departamento de Ciências da Terra da Universidade de Coimbra, Rua Sílvio Lima, 3030-790, Coimbra, Portugal; zepallac@gmail.com

(2) CITEUC - Centro de Investigação da Terra e do Espaço da Universidade de Coimbra, Portugal.

(3) Agrupamento de Escolas da Guia, Escola Básica e Secundária da Guia, Rua Fundadores do Colégio, 3105-075 Guia, Portugal; ricardojchp@gmail.com

(4) MARE - Marine and Environmental Sciences Centre, Portugal; pdinis@dct.uc.pt 
cipais esteios da Zooarqueologia, não se devendo a sua importância relativa distanciar demasiado da que tradicionalmente se atribui aos vertebrados. $\mathrm{Na}$ realidade, os seus estudos tafonómico e taxonómico podem proporcionar dados significativos sobre hábitos e práticas de recoleção e de alimentação, a par de costumes e vivências de indivíduos, comunidades e culturas, desde o Paleolítico. Dado um certo carácter interdisciplinar com a Paleontologia e a Zoologia (Fig. 1), são também numerosos os dados de índole taxonómica, ecológica, biogeográfica e paleoclimática que se encontram subjacentes a determinadas espécies ou associações de moluscos, cuja tipologia nos fornece indicações preciosas sobre a envolvente ambiental local/regional e respetivas biocenoses.

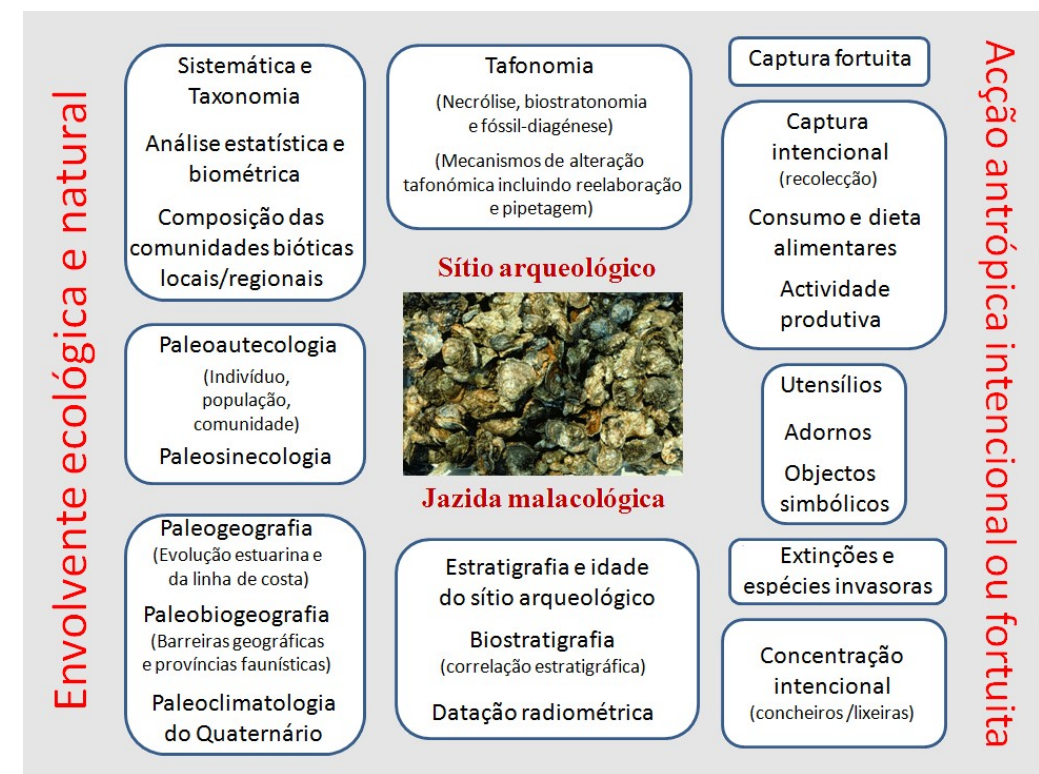

Fig. 1. Universo temático e interdisciplinar da Zooarqueologia aplicada a moluscos.

Fig. 1. Thematic and interdisciplinary universe of Zooarchaeology applied to molluscs.

Considerando a importância crescente que os estudos de Zooarqueologia de moluscos têm vindo a registar nas últimas duas décadas, no seguimento de uma consciencialização gradual que a comunidade arqueológica portuguesa tem tomado para a necessidade de complementar a interpretação de artefactos e seus contextos, com dados remanescentes de espólios de origem biológica, pretende-se analisar, nas páginas seguintes, alguns dos principais aspetos envolvidos neste tipo de análises multidisciplinares, tendo por base as perspetivas do paleontólogo e do biólogo, o primeiro mais atento à dimensão tempo e aos percursos da fossilização, o outro desde sempre motivado pelos ecossistemas e pela distribuição dos organismos.

\section{O ESPAÇO E A ENVOLVENTE BIÓTICA}

Os espólios malacológicos provenientes de contextos arqueológicos portugueses são bastante diversos quanto à sua composição taxonómica, reflexo das envolventes física e biótica locais, em conjugação com outros fatores condicionantes. Entre estes podem-se enumerar: (1) o percurso biostratonómico pré ou sindeposicional e consequente autoctonia ou alóctonia (FERNANDEZLÓPEZ 1999); (2) a ação antrópica, intencional ou fortuita, subjacente à acumulação ou concentração dos restos esqueléticos; (3) as alterações postdeposicionais devidas à intervenção de agentes bióticos, físicos ou químicos, estes últimos ligados à evolução diagenética do próprio depósito arqueológico.

Enquanto condicionante primordial que influência tal diversidade taxonómica, a envolvente biótica local/regional presente ao tempo da génese do depósito passa, necessariamente, por um universo de várias centenas de espécies de moluscos integrantes das comunidades marinhas litorais, a par das de paleoambientes de transição, com características salobras, e das de meios dulçaquícolas e terrestres.

Para além de certas exceções relacionadas com (1) migrações ou extinções originadas pela evolução paleoclimática ou por transformações no espaço físico, (2) a colonização do meio por espécies invasoras, (3) a introdução de espécies comestíveis, ou (4) o uso de conchas exóticas (CALLAPEZ et al. 2007), as espécies encontradas em contextos arqueológicos portugueses são análogas às que hoje formam a malacofauna marinha e terrestre do território. Deste modo, considera-se ser esta a base operacional dos estudos de moluscos portugueses, aplicados a contextos arqueológicos do Plistocénico e Holocénico (Fig. 2). 


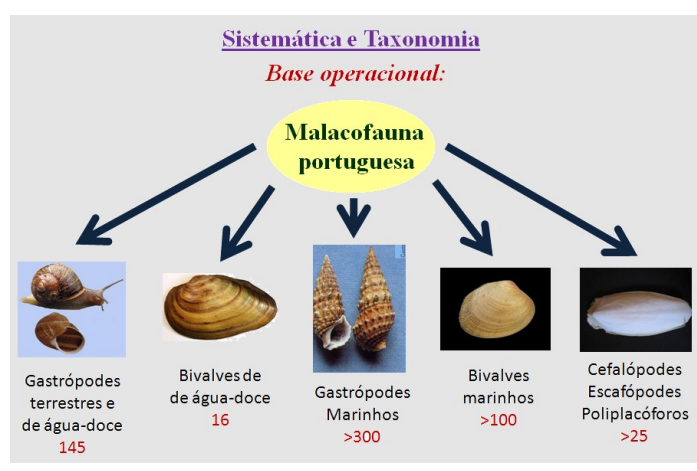

Fig. 2. Base operacional dos estudos de Zooarqueologia de moluscos em Portugal.

Fig. 2. Operational basis of the zooarchaeological studies of molluscs in Portugal.

As características desta fauna são hoje bem conhecidas, por força de nos encontrarmos no litoral europeu, objeto de grande número de publicações. Entre estas e para Portugal, são incontornáveis os trabalhos de Augusto Nobre (1931, 1936, 1941), hoje raros, mas excelentes pela sua exaustividade, apesar de carecerem de atualizações taxonómicas. Mais recentemente merecem referência, entre outras, as monografias e guias de Tebble (1976), Rolán (1984), Rolán et al. (1989), Poppe \& Goto (1991, 1993), Saldanha (1997), Rodríguez \& Sánchez (1997), Macedo \& Borges (1999) e Valente (2010b).

Quanto à sistematização de categorias taxonómicas superiores ao género, são de consulta necessária as monografias de Wenz (1938-44) e
Moore (1969). Não obstante, as últimas atualizações desenvolvidas mais de acordo com a filogenia das classes Gastropoda e Bivalvia respeitam, respetivamente, aos estudos de Bouchet et al. (2005) e Bieler et al. (2010).

\subsection{Moluscos terrestres e de água-doce}

A malacofauna terrestre e dulçaquícola é, comparativamente, menos diversificada do que a sua congénere marinha, sendo escassas as espécies com historial de uso alimentar, ou recolhidas para fins decorativos ou outros. $\mathrm{Na}$ generalidade os moluscos terrestres são habitantes autóctones dos sítios arqueológicos, conferindo-nos dados sobre as paleofaunas locais/regionais e seu possível espectro de variação temporal (CALLAPEZ 2002, 2003, 2014; Carvalho et al., 2015) (Fig. 3).

A taxonomia tradicional baseada em Nobre (1930, 1940), a par de outros trabalhos de menor extensão, passou por uma profunda revisão na última década e meia, destacando-se os estudos de Matos (1993a, 1993b, 1994, 2004), Holyoak \& Holyoak (2010, 2012, 2015) e Holyoak et al. (2012), a par de atlas sobre bivalves dulçaquícolas (REIS 2006) e de gastrópodes terrestes e aquáticos (Matos, 2014). Como base bibliográfica para o estudo da fauna portuguesa, são também de relevar, entre outras, as monografias de Kerney \& Cameron (1979) e Falkner et al. (2002). Quanto à sistematização de categorias superiores ao género, a obra de Zilch (1960) constitui um excelente suporte de trabalho.

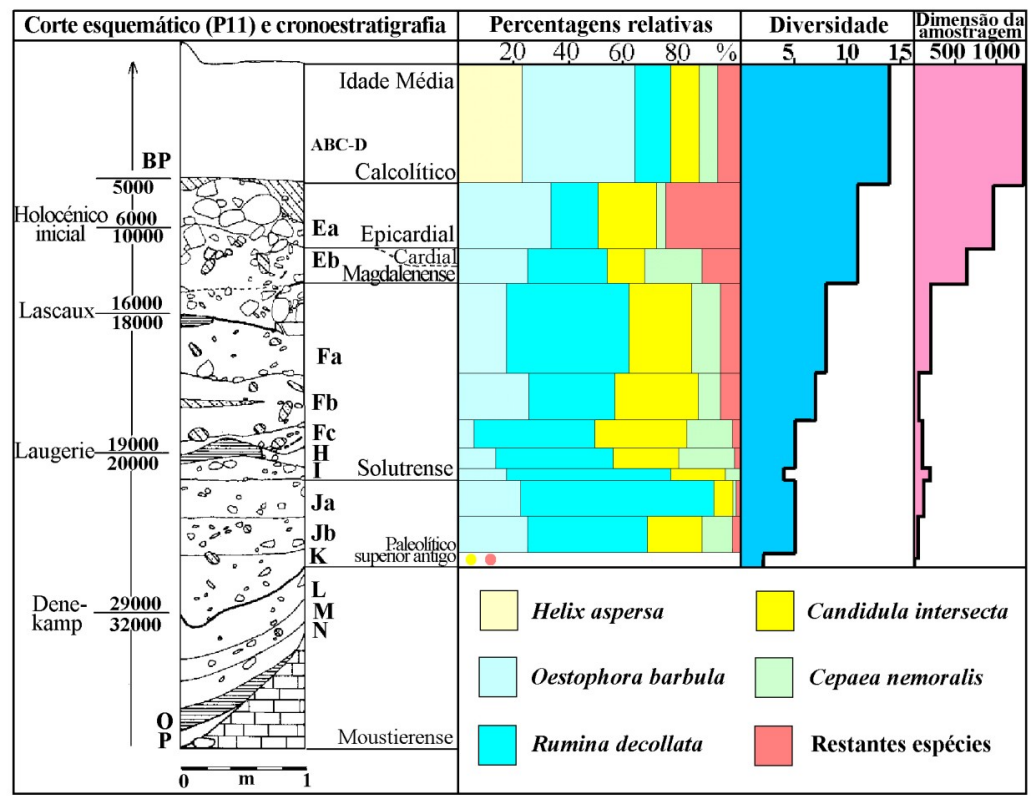

Fig. 3. Exemplo de análise quantitativa de moluscos terrestres em contexto com estratigrafia bem estabelecida e seu espectro de variação temporal. Gruta do Caldeirão, Tomar (segundo CALLAPEZ 2002; corte segundo ZILHÃ̃ 1997).

Fig. 3. Example of quantitative analysis of terrestrial molluscs contextualized with a well- established stratigraphy and its temporal variation spectrum. Caldeirão cave, Tomar, Portugal (CALLAPEZ 2002; section adapted from ZILHÃO 1997). 


\section{ASPETOS METODOLÓGICOS}

O estudo de acervos malacológicos presentes em arqueossítios pressupõe um faseamento que se inicia com o estudo in loco do contexto estratigráfico respetivo, através do qual se recolhem dados acerca da geometria do depósito e sobre a disposição espacial, orientação e densidade dos macrorrestos, os quais são determinantes para a compreensão dos processos envolvidos na formação da jazida.

Seguem-se a recolha, preparação e separação taxonómica dos macrorrestos conquiológicos, com vista à identificação dos taxa representados, à leitura e ordenamento dos mecanismos de alteração tafonómica neles intervenientes e à quantificação e tratamento estatístico da amostragem. Não obstante, o estado de preservação nem sempre facilita, ou possibilita, uma identificação taxonómica precisa, colocando dificuldades acrescidas às etapas seguintes. Entre outros fatores, contribuem para essa limitação o tipo de alteração biostratonómica das conchas originais, o seu grau de fragmentação e as dimensões dos fragmentos daí resultantes, muitos deles desprovidos de partes diagnósticas das espécies. Também os processos de alteração tafonómica pós-enterramento podem, aqui, ser particularmente influentes. Neste âmbito, é de grande utilidade o acesso a coleções comparativas com moluscos atuais, disponíveis nalguns museus portugueses ou através de particulares.

\subsection{Importância dos estudos de tafonomia}

A avaliação das condições de formação de um depósito arqueológico preservado com estratigrafia, assim como dos processos e agentes naturais ou antrópicos que levaram à acumulação de restos de natureza animal ou vegetal, em conjunto com os artefactos propriamente ditos, consiste numa das etapas fundamentais do estudo e interpretação de arqueossítios (e.g. ClAASSEN 1998; LANDON 2005). As análises tafonómicas, embora desenvolvidas inicialmente no âmbito da Paleontologia (tafonomia e fossilização), alargaram-se nas últimas décadas aos contextos arqueológicos, facto que faz sentido, dada a componente interdisciplinar de bioarqueologia que estes pressupõem (e.g. LYMAN, 1987; CLAASSEN 2000; GUTIÉRREZ ZUGASTI 2008).

No caso dos moluscos, este tipo de análises envolve não só uma apreciação da génese, geometria e sequenciação temporal do depósito, mas também a história tafonómica inerente a cada macrorresto, ou seja, a reconstituição do percurso por ele efetuado desde o momento da sua disponibilização no meio (por morte natural do organismo ou intervenção humana) até à sua inclusão no depósito e à atuação de alterações diagenéticas posteriores. $\mathrm{Na}$ primeira etapa, designada de biostratonómica, interatuam numerosos mecanismos de alteração tafo- nómica, construtivos ou destrutivos, resultantes de agentes físicos, químicos e/ou biológicos (FERNANDEZ-LÓPEZ 1999). Por sua vez, a avaliação do tipo de intervenção humana, se ativa ou passiva e em que medida esta influenciou as características do depósito e a natureza dos macrorrestos acumulados, também é de importância fundamental.

A heterogeneidade da tafonomia dos macrorrestos de moluscos presentes em depósitos arqueológicos é, por demais, evidente quando se consideram exemplos de enchimentos sedimentares de grutas e outras cavidades naturais ou artificiais (CAllaPeZ 2000). Na realidade, a amálgama de conchas ou de outros restos de invertebrados poderá resultar de:

(a) Atividade animal contemporânea da formação do depósito;

- Transporte ativo, ligado ou não a predação, por parte de mamíferos, répteis ou aves;

- Transporte passivo de macrorrestos com peso e dimensões reduzidas (ex: pequenas conchas ou fragmentos presos ao corpo de animais);

- Presença de moluscos terrestres, habitantes naturais do arqueossítio;

(b) Reelaboração tafonómica de restos esqueléticos (FERNANDEZ-LÓPEZ 1999);

- Reelaboração de conchas fósseis provenientes da desagregação mecânica das paredes ou teto da cavidade;

- Contaminação a partir do exterior, por inundação ou transporte gravítico (ex: a partir de depósitos com conchas existentes perto da abertura da cavidade);

(c) Pipetagem de conchas mais modernas, inerente à bioturbação da estratigrafia original do depósito arqueológico por parte do Homem ou de animais escavadores (ex: toupeira, texugo, coelho).

(d) Atividade humana contemporânea da formação do depósito;

- Transporte passivo de macrorrestos de pequenas dimensões;

- Concentrações de "restos de cozinha";

- Aproveitamento de conchas como utensílios;

- Utilização de conchas, simples ou modificadas intencionalmente, como matéria-prima para a confeção de elementos de adorno.

\subsection{Quantificação e análise estatística}

Este tipo de estudo deve ser empreendido quando o acervo malacológico amostrado a partir do arqueossítio é numeroso e permite, por outro lado, a identificação taxonómica dos restos esqueléticos (elementos) e a sua contabilização por espécie, quadrícula e nível estratigráfico. Para além da quantificação das abundâncias relativas de diferentes 
taxa, também se podem efetuar estudos de natureza biométrica (Fig. 4), ou ainda de morfometria, nos quais se comparam forma e dimensão, como, por exemplo, em Cabral \& Silva (2003), na análise morfométrica de lapas em contexto da Idade do Ferro.

Em alternativa é comum a elaboração de tabelas semiquantitativas ou, simplesmente, que expressem a presença/ausência de cada taxon por nível e/ou quadrícula, de acordo com o levantamento dos trabalhos arqueológicos.

Seguindo-se a via quantitativa e perante taxas de fragmentação significativas das conchas, é necessário proceder à reconversão do número de elementos em número estimado de espécimes, segundo critérios coerentes. Como exemplo, nos moluscos bivalves é vulgar a contabilização da valva mais numerosa de cada espécie, incluindo os fragmentos umbonais com charneira e dentição (CLASSEN 1998; SILVA 2000; GONÇALVES et al. 2007).

As análises uni e bivariantes têm vindo a ser as mais utilizadas em trabalhos de Zooarqueologia portuguesa (Fig. 4). Não obstante, quando a amostragem o permite, é possível o tratamento matemático de matrizes multidimensionais, em que a repartição quantitativa das diferentes espécies é cruzada com a das quadrículas e/ou a dos níveis estratigráficos, dando-se assim conta de possíveis tendências subjacentes à heterogeneidade dos depósitos arqueológicos.

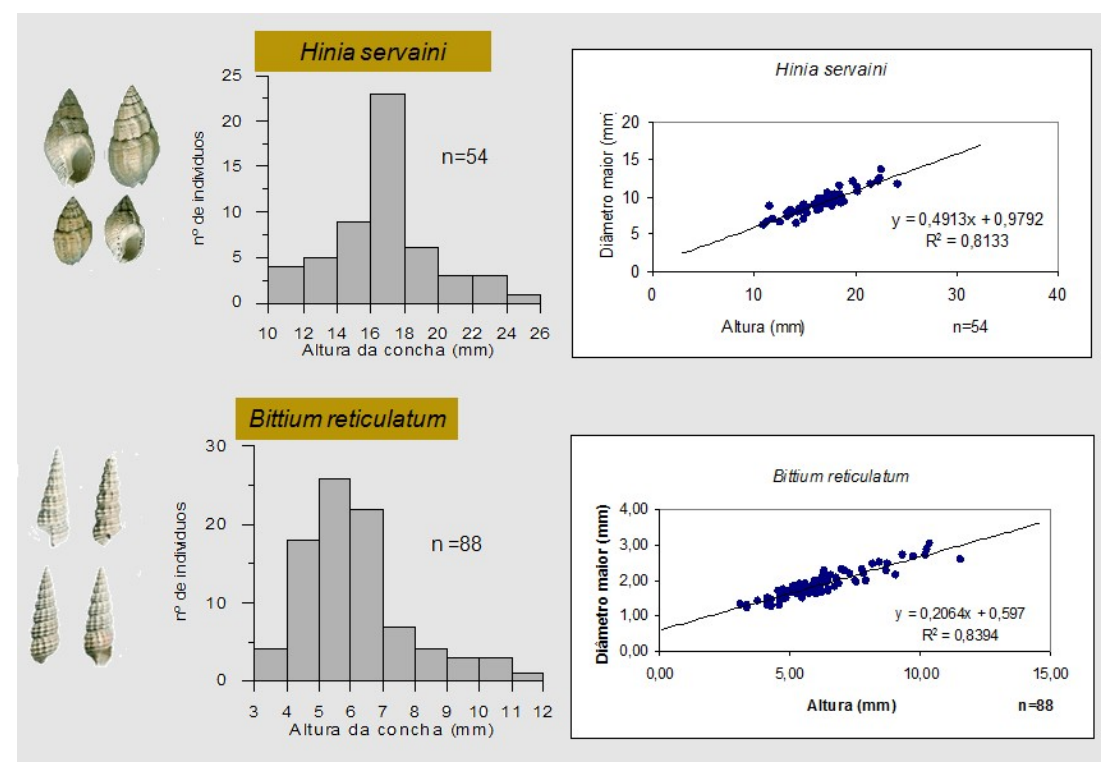

Fig. 4. Exemplo de análise quantitativa univariante e bivariante aplicada a duas espécies de moluscos marinhos holocénicos, provenientes de lodos arenosos lagunares da praia de Leirosa (Figueira da Foz), ca. 2500 BP (Segundo PAREDES et al. 2006).

Fig. 4. Example of univariate and bivariate quantitative analysis of two Holocene species of marine molluscs collected from lagoonal sandy muds in Leirosa beach (Figueira da Foz, Portugal), ca. 2500 BP (PAREDES et al. 2006).

\section{OS MOLUSCOS E O MEIO FÍSICO E BIÓTICO}

Embora não sendo o tipo de dados mais imediatistas e relevantes para a caracterização do contexto arqueológico per si, as informações de natureza paleobiológica inerentes às espécies ou associações de moluscos recolhidas durante a intervenção, constituem um documento inestimável sobre aspetos do meio físico e das comunidades bióticas locais ou regionais, naquilo que atrás se designou por envolvente ecológica (Fig. 1). Neste tipo de análise e porque falamos de espécies com representantes atuais, é fundamental a aplicação de critérios próprios do Uniformitarismo taxonómico, fundamentados na observação e no conhecimento diretos da ecologia de cada tipo de molusco e do seu papel no ecossistema.

\subsection{Reconstituições paleoecológicas e paleoambientais}

As análises de âmbito autecológico (indivíduos e paleopopulações de uma dada espécie) ou sinecológico (paleocomunidades) podem tornar-se bastante exaustivas quando as amostragens são numerosas e estatisticamente representativas, atingindo diversidades da ordem das dezenas de espécies. Para além dos moluscos, devem ser integradas com dados relativos a outros tipos de invertebrados bentónicos, comuns em concentrações de concheiros e de lixeiras, tais como briozoários, anelídeos poliquetas, crustáceos cirrípedes e decápodes, entre outros. Numa perspetiva mais abrangente, devem também ser tidos em conta dados relativos a macro e microrrestos de plantas, 
vertebrados, foraminíferos e quaisquer outros grupos taxonómicos presentes, com vista a aprofundar o detalhe dos estudos e das reconstituições deles derivadas.

Dado que este tipo de concentrações é marcadamente alóctone, é comum a coexistência de espécies pertencentes a diferentes biótopos ou, quando de meio litoral, representativas de diferentes patamares de zonação batimétrica. Como metodologia de trabalho, é usual a construção de tabelas de síntese, com dados para cada espécie e relativos a: (1) tipo trófico, (2) mobilidade/tipo de fixação, (3) posição no substrato, (4) tipo de substrato, (5) zonação batimétrica, e (6) tolerância à salinidade. Em paralelo é, por vezes, viável o delinear de um perfil ecológico, na aceção de Morton et al. (1998) (Fig. 5).
Para além de contribuírem para uma reconstituição espácio-temporal da envolvente ecológica e natural do arqueossítio, estas sínteses permitem, também, uma visão mais efetiva das características dos locais ou territórios de recoleção, motivadoras da preferência por determinadas espécies comestíveis, em detrimento de outras, como o demonstram diversos estudos portugueses que já desenvolveram esta problemática (e.g. SILVA et al. 1986; SILVA \& SOARES 1993, 1997, 1998; NUÑO 1993; ANTUNES 1996; SILVA 1997, 2000; GONÇALVES 2006; GONÇALVES et al. 2007; CALLAPEZ 2007, 2011, 2012; VALENTE 2010a; COELHO \& CARDOSO 2010-2011; DETRY \& ARRUDA, 2012; DINIS \& ARIAS, 2012; VALENTE \& MARTINS, 2015).

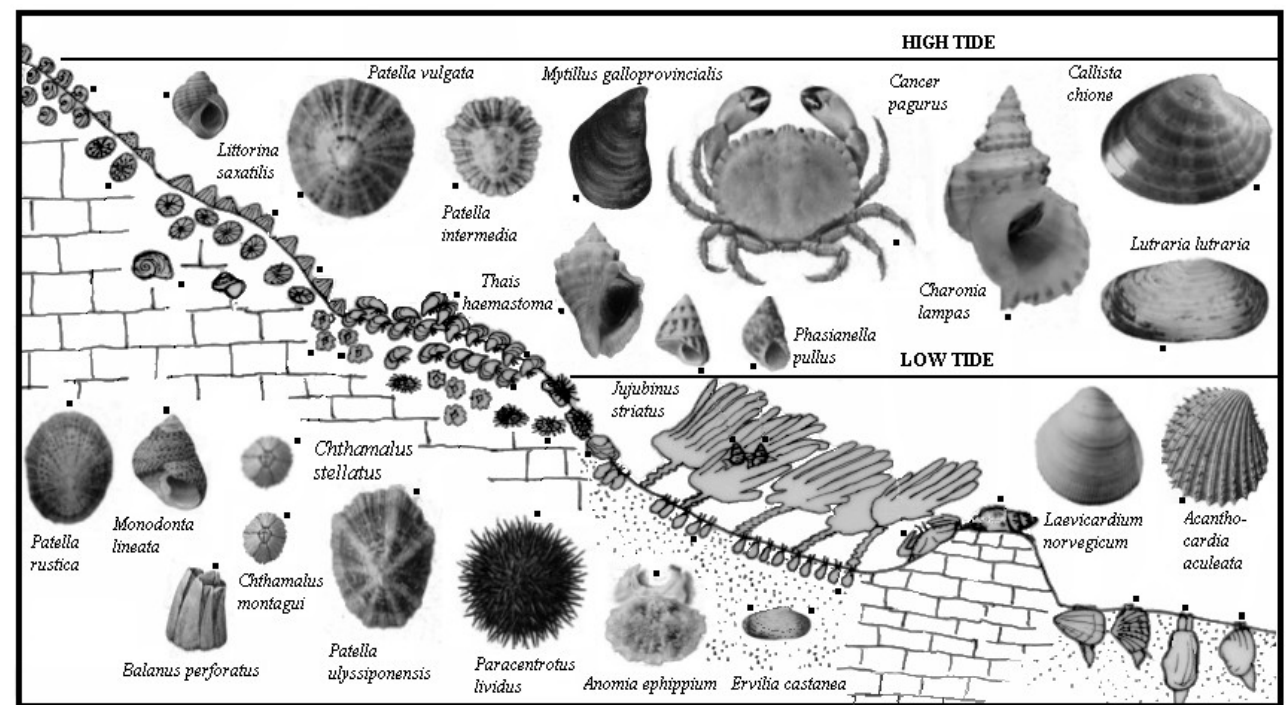

Fig. 5. Exemplo de perfil ecológico interpretativo, baseado no estudo dos macrorrestos de invertebrados do Paleolítico da Gruta da Figueira Brava (Portinho da Arrábida) (segundo CALLAPEZ 2000).

Fig. 5. Example of interpretive ecological section based on the study of invertebrate macroremains from the Paleolithic of Figueira Brava cave (Portinho da Arrábida, Portugal) (CALlaPEZ 2000).

\subsection{Dados biogeográficos e climáticos}

A costa ocidental portuguesa e o litoral algarvio situam-se numa posição de charneira face à dispersão da fauna temperada do litoral atlântico da Europa e das suas congéneres mediterrânica e norte -africana. Tendo em conta os sistemas de correntes oceânicas do domínio atlântico (LONGHURST 1988; VANTREPOTTE \& MÉLIN 2009) e a sua influência significativa na dispersão das espécies, as comunidades de moluscos marinhos do litoral de Portugal continental integram as biocenoses costeiras das regiões sob influência do Northeast Atlantic Subtropical Gyre (NASE), sendo que a grande maioria das espécies de bivalves e de gastrópodes apresentam áreas de repartição biogeográfica que se prolongam até ao Canal da Mancha ou ao Mar do Norte, já dentro dos limites do North Atlantic Drift (NADR).

As faunas presentes no litoral português patenteiam, deste modo, características marcada- mente temperadas, inserindo-se na província biogeográfica Lusitânica, com extensão até ao golfo da Biscaia e, para Sul, até ao litoral de Marrocos e da Mauritânia, onde confronta com a província de Transição Oeste Africana (SPALDING et al. 2007). Por sua vez, no litoral do Algarve são mais nítidas as influências da proximidade da província Mediterrânica e da costa ocidental de África, espaços que, pelas suas características físicas e bióticas, apresentam características diferenciadas nas associações de espécies de moluscos presentes.

Desta forma, é possível a ocorrência de episódios em que, quando um maior aquecimento sazonal das águas superficiais o permita, a dispersão larvar de espécies termófilas, típicas de contextos biogeográficos de mais baixa latitude, pode resultar em situações de colonização pontual no litoral português, ainda que com maior incidência no Algarve. Constituem exemplos recentes, por nós confirmados no atual litoral desta região, os gastrópodes Naticarius hebraeus (MARTYN 1786), 
Demoulia obtusata (LINK 1807) e Vexillum tricolor (GMELIN 1791), os quais contribuem para ampliar a área de repartição biogeográfica conhecida destas espécies, graças à instalação de populações viáveis em áreas anteriormente inóspitas.

A mesma dispersão larvar, se associada a oscilações climáticas de maior amplitude, pode dar origem a populações estáveis, naquilo que corresponde a uma extensão migratória e consequente ampliação da área de repartição biogeográfica das espécies envolvidas. A presença destes "warm guests" ou, por contraposição, de espécies de ambientes mais frios, fica, por vezes, registada em depósitos plistocénicos ou holocénicos, associados ou não a contextos arqueológicos (CALLAPEZ et al. 2012).

Numa perspetiva de longo termo e na aparente ausência das tradicionais "faunas quentes" e "faunas frias" que tão bem caracterizam depósitos de praia do Plistocénico da Europa mediterrânica, são mais relevantes em Portugal as introduções faunísticas resultantes da evolução climática pósWürm e ligadas à transgressão "flandriana" que transformou profundamente o nosso litoral. Neste contexto, o aquecimento progressivo das águas superficiais terá propiciado a dispersão, ao longo do litoral oeste da Ibéria, de diversas espécies outrora já existentes no litoral português.

Uma das evidências mais curiosas prende-se com a população relíquia de Naticarius stercusmuscarum (GMELIN 1791) outrora existente no Mar da Palha, no estuário do rio Tejo, a qual teria proliferado durante um desses intervalos de maior aquecimento climático, tendo sido recolhidos exemplares, por um dos autores, em dragagens de areias holocénicas, no Lavradio. Esta espécie de naticídeo restringe-se, presentemente, ao Mar Mediterrâneo (POPPE \& GOTO 1991).

Em contextos ligados a concheiros do Mesolítico e do Neolítico Antigo do Sul de Portugal, mas também em concentrações de lixeira com cronologias até finais do Período Islâmico, é comum a ocorrência de grandes quantidades de conchas de púrpura [Stramonita haemastoma (LINNAEUS 1767)], de burriés, de mexilhão e de várias espécies de lapa, nem sempre de fácil classificação. A primeira destas espécies, ou, na realidade, um complexo de várias espécies com morfologias bastante próximas entre si (VERMEIJ 2001), é anfiatlântica de águas temperadas/quentes e terá (?re) colonizado as áreas rochosas do litoral português após o final do Würm, pelo menos até ao Cabo da Roca, conforme fragmentos de conchas encontradas, por um dos autores, em depósitos superficiais holocénicos assentes sobre arribas a norte da praia do Abano. Encontra-se hoje em acentuada regressão, muito por força da pressão antrópica e de séculos de captura excessiva (Fig. 6a).

Embora confundido, muito possivelmente, com o mexilhão-comum [Mytilus edulis (LINNAEUS 1758)], aquando da identificação taxonómica de conchas provenientes de contextos arqueológicos do Algarve e do Sudoeste Alentejano, o grande mitilídeo comestível do litoral oeste-africano [Perna perna (LiNNAEUS 1758)] terá acompanhado a espécie anterior na colonização do litoral Sul de Portugal (Fig. 6b). Este bivalve foi por nós referenciado no Neolítico Antigo de Padrão 1 (Vila do Bispo) e é frequente no concheiro da Arrifana (Aljezur), este último de finais do Período Islâmico (GOMES \& GOMES 2005; CALLAPEZ 2007, 2011; CALLAPEZ et al. 2012).

Também confundida graças à convergência morfológica da sua concha com as das lapas (Patella spp.), o gastrópode pulmonado Siphonaria pectinata (LINNAEUS 1758) representa outro exemplo de espécie oeste-africana, frequente em depósitos de importância arqueológica e que terá colonizado parte do litoral português após o final do Würm (Fig. 6c). Não obstante, está espécie não é comestível, pois segrega um muco tóxico de paladar desagradável (OCAÑA 2003), pelo que a sua presença em contextos de restos de cozinha terá resultado de recolha fortuita, por confusão com as espécies de lapa vulgarmente existentes nos mesmos biótopos rochosos litorais.

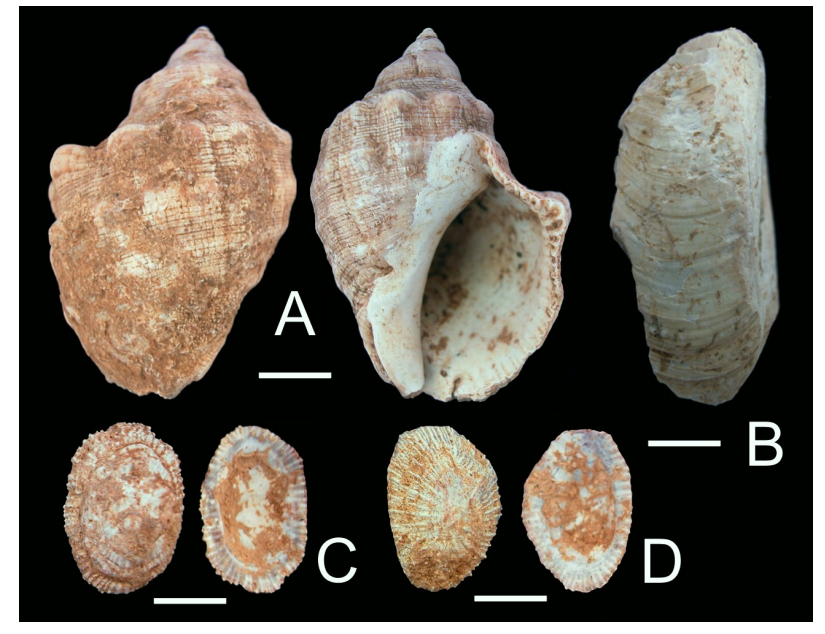

Fig. 6. Espécies marinhas oeste-africanas, mediterrânicas e/ou anfiatlânticas, colonizadoras do litoral português durante o aquecimento climático pós-Würm. A - Stramonita haemastoma (Linnaeus, 1767); B - Perna perna (Linnaeus, 1758); C - Siphonaria pectinata (Linnaeus, 1758). Espécimes provenientes do sítio arqueossítio do Neolítico Antigo de "Padrão 1" (Vila do Bispo). Escala da barra: $1 \mathrm{~cm}$.

Fig. 6. West African, Mediterranean and/or anphiatlantic marine species that colonised the Portuguese coast during the post-Würm global warming. A - Stramonita haemastoma (Linnaeus, 1767); B - Perna perna (Linnaeus, 1758); C Siphonaria pectinata (Linnaeus, 1758). Specimens from the Ancient Neolithic site of "Padrão 1" (Vila do Bispo, South Portugal). Scale bar: $1 \mathrm{~cm}$. 


\section{OS MOLUSCOS E A INTERVENCỸ̃O HUMANA}

Em paralelo com uma análise vocacionada para a reconstituição de aspetos do meio físico e das comunidades bióticas existentes na envolvente do contexto arqueológico, ao tempo da génese do depósito, os estudos zooarqueológicos baseados em associações de moluscos devem ser dirigidos de modo a diferenciar processos puramente naturais, de percursos em que teve lugar a intervenção humana, intencional ou passiva. Nesta etapa, as metodologias decorrentes das análises sistemática e tafonómica são fundamentais. Com efeito, o volume de dados de natureza taxonómica, biostratonómica, paleobiológica e biogeográfica que encerra uma concentração de conchas subfósseis pode ser bastante útil para a formulação de hipóteses sobre hábitos de recoleção e de alimentação, trocas e contactos entre grupos ou populações, ou ainda atividades produtivas e percursos e rotas comerciais, dentro de uma área geográfica ou regiões limítrofes.

Desta forma, entre as evidências de intervenção humana intencional registadas a partir da interpretação de restos malacológicos, há que considerar, sobretudo, as que estão ligadas à captura e processamento de espécies comestíveis, hábitos e dietas alimentares. Outras ainda, estão relacionadas com o aproveitamento funcional de conchas para utensílios de uso quotidiano, ou ainda, com aspetos de ordem estética e cultural, motivadores da seleção de formas, cores e padrões para a criação e elaboração de objetos de adorno (e.g. Silva \& CABrita 1966; FERreira \& Roche 1980; CHAUVIÈRE 2002; VANHAEREN \& D'ERRICO 2002; ZILHÃO 1992, 1997; ZILHÃO et al. 2010; TÁTÁ et al. 2013; DEAN \& CARVALHO 2014).

\subsection{Espécies comestíveis e recursos ali- mentares}

Das muitas espécies de moluscos comestíveis utilizadas regularmente como recurso alimentar e, como tal, encontradas em contextos de concheiro ou de lixeira (e.g. FERREIRA 1956; SILVA \& CABRITA 1966; MoreNO-NuÑo 1993; SILVA 1996; SILVA \& SOARES 1993, 1997, 1998; CARVALHO \& VALENTE 2005; GONÇALVES 2006; GONÇALVES et al. 2007; CARDOSO \& COELHO 2010; CALLAPEZ 2007, 2011, 2012; MARTINS 2013; VALENTE \& MARTINS 2015), a maior parte provém de meio litoral, refletindo a exploração metódica de áreas costeiras rochosas, de praias e baixios arenosos, ou ainda de estuários ou lagunas com substratos mais finos e confinados. Esta exploração, quer sob a forma de simples recoleção incidente sobre os stocks naturais disponíveis, quer derivada de uma produção controlada em viveiros, desde sempre obedeceu à periodicidade dos ciclos de maré, à sazonalidade e à própria dinâmica populacional e ciclos biológicos das espécies de moluscos. Neste quadro, o cultivo de certos bivalves em meio lagunar/estuarino, incluindo a amêijoa Ruditapes decussatus (LinNAEUS, 1758) e a ostra comum Ostrea edulis LinNAEUS, 1758, deverá remontar à Pré-História Recente, embora com forte incremento nas épocas Romana e Islâmica.

Também a partir de finais da Pré-História, algumas espécies de meio infralitoral terão começado a ser consumidas regularmente, na qualidade de subprodutos da pesca artesanal com redes que varejavam substratos arenosos ou rochosos pouco profundos. Encontram-se, entre estas, a buzina [Charonia lampas (LINNAEUS, 1758)], a canilha [Bolinus brandaris (LINNAEUS, 1758)], as castanholas [Glycymeris glycymeris (LINNAEUS, 1758) e G. nummaria (LINNAEUS, 1758) ] e a vieira [Pecten maximus (LinNAEUs, 1758)].

O consumo de todas estas espécies no passado terá beneficiado de um litoral ainda escassamente explorado, como o comprovam a abundância e as dimensões de algumas delas [e.g. Stramonita haemastoma (LiNNAEUS, 1767), Patella vulgata LinNAEUS, 1758], hoje localmente extintas, raras ou, aparentemente, com menor desenvolvimento biométrico, mas bastante importantes em contextos arqueológicos até à Idade Média (CAllapez 2007, 2011).

O processamento in loco destes moluscos para fins alimentares é, por sua vez, evidenciado por alterações tafonómicas das conchas que não seriam possíveis ou prováveis através de processos naturais. Estas envolvem: (1) a desarticulação intencional de bivalves, suscetível de se identificar quando incide, preferencialmente, sobre valvas de espécies comestíveis, sem vestígios biostratonómicos de abrasão, bioerosão e/ou fixação de epizoários nas suas partes internas; (2) a fracturação ou perfuração padronizadas de conchas de gastrópodes por percussão dirigida e (3) a calcinação de conchas, muitas vezes ligada a estruturas de combustão.

Quanto aos moluscos terrestres, as evidências sobre o seu consumo regular remontam, seguramente ao Período Romano. Não obstante, é comum a presença de caracóis comestíveis em contextos arqueológicos mais antigos, pelo que existe a possibilidade de que estas tenham sido utilizadas como alimento (LUBELL 2004). Entre outras espécies, a "caracoleta" Cornu aspersa (LinNAEUs, 1758), hoje muito abundante em todo o território, terá sido uma introdução tardia na fauna portuguesa, relacionada, muito possivelmente, com a Romanização, conforme o comprovam dados de locais com estratigrafia, entre os quais os níveis dos "Tufos de Condeixa" e o enchimento da Gruta do Caldeirão (CAllapez 1992, 2002).

Já do final do Período Islâmico, foram encontradas evidências, em contexto de lixeira, de conchas de Theba pisana (MÜLLER, 1774) com perfurações de alfinete, ou de objeto com função equiva- 
lente, utilizado para a extração do animal cozinhado (CALlapez 2012). Esta espécie é, ainda hoje, o pulmonado terrestre mais consumido na gastronomia tradicional do Sul de Portugal.

\subsection{Utilidade funcional}

São também expressivos os exemplos ligados ao uso de conchas de moluscos marinhos, recuando certas práticas ainda atuais a épocas remotas. Entre estes considera-se a utilização de valvas de Glycymeris glycymeris (LINNAEUS, 1758) ou de G. nummaria (LINNAEUS, 1758) como prováveis pesos de pesca, facto que poderá explicar a presença de valvas grandes e espessas destas espécies, quase sempre com facetas umbonais de abrasão biostratonómica, em diversos espólios malacológicos dos Períodos Romano, Islâmico, ou posteriores (GONÇALVES et al. 2007). Este facto pressupõe uma recolha seletiva de valvas destas espécies em cordões arenosos do litoral algarvio ou da costa ocidental onde são, por vezes, bastante comuns.

De igual modo, são conhecidos exemplos de valvas de Panopea glycymeris (BORN, 1778) e de valvas côncavas de Pecten maximus (LINNAEus, 1758), suscetíveis de terem sido aproveitadas como recipientes (CALLAPEZ 2011). A primeira destas espécies é o maior bivalve da malacofauna portuguesa, ocorrendo raramente em detritos de maré, na sequência de temporais de inverno. Ainda hoje se regista a sua utilização no seio das comunidades de pescadores da Ria Formosa.

\subsection{Interesse estético e cultural}

A ocorrência de conchas utilizadas como elementos de adorno em contextos funerários de grutas e abrigos situados em território português é conhecida desde o Paleolítico Superior, também com bastante incidência no Neolítico e Calcolítico (e.g. Silva \& CABRITA 1966; FERREIRA \& RochE 1980; CHAUVIÈRE 2002; VANHAEREN \& D'ERRICO 2002; ZILHÃo et al. 2010; TÁTÁ et al. 2013; DEAN \& CARVAlHo 2014; CARVAlHo et al. 2015). Do número crescente de exemplos conhecidos, salientamos o acervo da Gruta do Caldeirão, situada perto de Tomar (ZILHÃO 1992; CHAUVIÈRE 2002), no qual se encontrou um número apreciável de espécimes perfurados do neritídeo dulçaquícola Theodoxus fluviatilis (LinNAEUS, 1758), a par de conchas de Unio pictorum (LinNAEUS, 1758), Glycymeris glycymeris (LINNAEUS, 1758), Mytilus edulis LINNAEUS, 1758 e Nassarius pfeifferi (PhilipPI, 1844) (CALlaPeZ 1992).

As valvas de Glycymeris glycymeris (LiNNAEUS, 1758) e de G. nummaria (LiNNAEUS, 1758) com perfurações naturais devidas a abrasão biostratonómica também não são raras em sítios mesolíticos ou neolíticos (CARVALHO et al. 2010; CAllapez 2011). A diversidade de conchas com perfurações intencionais, recolhidas nestes contextos, também se estende a muitos outros tipos de gastrópodes e bivalves, incluindo espécimes pertencentes aos géneros Natica e Trivia (VALENTE 2010a; DeAn \& CARVAlHo 2014), Stramonita [Thais] (ZBYSZEWSKI et al. 1981; CARVALHO \& CARDOso 2003) e Cerastoderma (SousA 1998).

Numa primeira perspetiva sobre estes e outros acervos malacológicos, as espécies presentes e sua frequência relativa parecem resultar da conjugação da seguinte ordem de fatores: (1) proximidade entre os habitats e locais de recolha e o de ocupação, (2) inserção do local de recolha no território de recoleção, (3) acessibilidade e grau de abundância no biótopo local, (4) presença de facetas de desgaste ou de perfurações naturais nos espécimes, (5) robustez das conchas e sua resistência a perfurações intencionais e (6) a própria sensibilidade estética do coletor perante formas, cores e padrões.

Da recolha e processamento dos materiais malacológicos ao processo criativo, à confeção e utilização destes elementos de adorno, de importância simbólica, votivos ou de estatuto, comuns na nossa herança arqueológica, medeia um universo de culturas e de significados místicos que cabe ao arqueólogo tentar interpretar, num desafio nem sempre fácil.

\section{PERSPETIVAS PRESENTES E FUTURAS}

A motivação do presente texto advém da necessidade sentida por uma fração crescente dos arqueólogos portugueses, no sentido de uma maior e mais metódica interdisciplinaridade dos seus estudos com outras áreas transversais do conhecimento científico, capazes de incrementarem significativamente o volume de dados recolhidos e o detalhe e abrangência das interpretações decorrentes dos contextos arqueológicos.

Quanto à Zooarqueologia de moluscos ou Arqueomalacologia, no dizer de Garcia et al. (2010), está-se perante uma área já bem estabelecida nos estudos de Arqueologia portugueses, em que o carácter de novidade há muito se desvaneceu. Não obstante, é para alguns ainda um recurso menor, subordinado às vicissitudes da dimensão de amostragens recolhidas por arrastamento e ao sentido de oportunidade de alguém que possa aparecer para as estudar, corroborando hipóteses já estabelecidas a partir do contexto e seus artefactos. Não nos parece ser esse o caminho a seguir, numa perspetiva futura em que será fundamental o incremento do número de investigadores na área da Zooarqueologia de invertebrados, a par de uma maior colaboração entre si e com os profissionais de Arqueologia. 


\section{DEDICATÓRIA E AGRADECIMENTOS}

Os autores dedicam este modesto contributo ao Professor Galopim de Carvalho, lembrando a sua longa e apaixonada entrega à causa da Paleontologia em Portugal. Agradecem, também, a dois revisores anónimos as análises críticas e construtivas do manuscrito, que em muito o enriqueceram e beneficiaram.

\section{BIBLIOGRAFIA}

ANTUNES, M.T. 1996. Alimentação de Origem Animal em Regime Islâmico - Alçaria Longa e Casa II da Alcáçova de Mértola. Arqueologia Medieval, 4: 267 $-276$

Bieler, R.; CARTer, J.G. \& CoAn, E.V. 2010. Classification of bivalve families. In: Bouchet, Ph.; Rocroi, J. -P.; Bieler, R.; Carter, J.G. \& Coan, E.V. 2010 (Eds.), Nomenclator of bivalve families. Malacologia, 52 (2): 1-184.

BORN I. VON 1778. Index rerum naturalium Musei Casarei Vindobonensis. Pars I.ma. Testacea. Verzeichniß der natürlichen Seltenheiten des $k$. $k$. Naturalien Cabinets zu Wien. Vindobonæ, Kraus.

Bouchet, P.; RocroI, J.-P.; FrÝdA, J.; HAUSdorF, B.; PONDER, W.; VAldÉs, Á. \& WARÉN, A. 2005. Classification and nomenclator of gastropod families. Malacologia, 47: 1-397.

BREUIL, H. \& ZBYSZEWSKI, G. 1947. Révision des industries mésolithiques de Muge et de Magos (Colletions des Services Géologiques de Portugal). Comunicações dos Serviços Geológicos de Portugal, 28: 149-196.

CABRAL, J.P. \& Silva, A.C.F. (2003). Morphometric analysis of limpets from an Iron-Age shell midden found in northwest Portugal. Journal of Archaeological Science, 30 (7): 817-829.

CAllapeZ, P.M. 1992. Moluscos terrestres das camadas A/B/ $\mathrm{C}-\mathrm{Eb}$ da Gruta do Caldeirão. In: Zilhão, J. (Coord.), Gruta do Caldeirão: O Neolítico Antigo. Trabalhos de Arqueologia, 6: 223-230 (apêndice C).

CAllapeZ, P.M. 2000. Upper Pleistocene marine invertebrates from Gruta da Figueira Brava (Arrábida, Portugal). In: Antunes, M.T. (Coord.), Last Neanderthals in Portugal, odontologic and other evidence. Memórias da Academia das Ciências de Lisboa, 38: 83-104.

CAllaPeZ, P.M. 2002. A malacofauna críptica da Gruta do Caldeirão (Tomar, Portugal) e as faunas de gastrópodes terrestres do Plistocénico superior e Holocénico da Estremadura portuguesa. Revista Portuguesa de Arqueologia, 5: 5-28.

CAllapez, P.M. 2003. Moluscos marinhos e fluviais do Paleolítico superior da Gruta do Caldeirão (Tomar, Portugal): evidências de ordem sistemática, paleobiológica e paleobiogeográfica. Revista Portuguesa de Arqueologia, 6: 5-15.

Callapez, P.M. 2007. Fauna Malacológica do ribāt da Arrifana - Análise preliminar. In: Gomes, R.V. \& Gomes, M.V. (Eds), Ribāt da Arifana. Cultura material e espiritualidade. Aljezur, Associação de Defesa do Património Histórico e Arqueológico de Aljezur: 87-90.

Callapez, P.M.; Balbino, A. \& Telles Antunes, M. 2007. Fauna malacológica moderna no Claustro da
Academia das Ciências de Lisboa. Memórias da Academia das Ciências de Lisboa, 43 (2) (2011): 435-450.

CALlapez, P.M. 2011. Estudo zooarqueológico dos invertebrados do Ribāt da Arrifana (Aljezur, Portugal). Sua relação com as comunidades marinhas litorais e com hábitos alimentares no algarve muculmano do século XII. In: Gomes, R.V.; Gomes, M.V. \& Tente, C. (Eds.), Cristãos e Muçulmanos na Idade Média Peninsular. Encontros e Desencontros. Lisboa, Instituto de Arqueologia e Paleociências das Universidades Nova e do Algarve: 165-186.

CALlaPeZ, P.M. 2012. Invertebrados: aspectos da malacofauna e do consumo de moluscos no Algarve muçulmano. In: Gomes, M.V. (Coord.), Silo Islâmico de Albufeira (Rua Henrique Calado). Lisboa, Instituto de Arqueologia e Paleociências e Autores: 85-95.

Callapez, P.M.; Danielsen, R. \& Castilho, A. 2012. Occurrences of the amphi-Atlantic brown musse Perna perna (Linnaeus, 1758) (Mollusca, Bivalvia) in South Portugal since the Atlantic "climatic optimum”. Estudos do Quaternário, APEQ, 8: 13-21.

Callapez, P.M. 2014. Terrestrial Gastropods. In: Carvalho, A.F. (Coord.), Bom Santo Cave (Lisbon) and the Middle Neolithic Societies of Southern Portugal. Promontoria Monográfica, 17: 45-61.

CARdoso, J.L. \& CoelHo, M.D. 2010. The marine malacological remains from the chalcolithic fortified settlement at Outeiro Redondo (Sesimbra): collection strategies used by a sedentary community from the 3rd millennium BC on the Portuguese coast. Zephyrus, 70: 85-111.

Carvalho, A.F. \& CARdoso, J.L. 2003. A estação do Neolítico antigo de Cabranosa (Sagres). Contribuição para o estudo da neolitização do Algarve. Trabalhos de Arqueologia, 25: 23-43.

Carvalho, A.F. \& Valente, M.J. 2005. Novos contextos conquíferos pré-históricos na Costa Vicentina. Atas do II Encontro de Arqueologia do Algarve. Xelb, 5: $9-26$

Carvalho, A.F.; Valente, M.J. \& Dean, R.M. 2010. O Mesolítico e o Neolítico antigo do concheiro da Rocha das Gaivotas (Sagres, Vila do Bispo). Xelb, 10: 39-53.

Carvalho, A.F.; Alves-Cardoso, F.; Gonçalves, D.; GranJa, R.; CARDOSO, J.L.; DEAN, R.M.; GIBAJA, J.F.; MASUCCI, M.A.; ARROYO-PARdo, E.; FERnÁNDeZ-Domínguez, E.; Petchey, F.; Price, T.D.; Mateus, J.E.; QueIroZ, P.F.; Callapez, P.M.; Pimenta, C. \& Regala, F.T. 2015. The Bom Santo Cave (Lisbon, Portugal): Catchment, Diet, and Patterns of Mobility of a Middle Neolithic Population. European Journal of Archaeology, 19 (1) (online ed.).

ChAuviÈRe, F. 2002. Industries et parures sur matières dures animales du Paléolithique supérieur de la grotte de Caldeirão (Tomar, Portugal). Revista Portuguesa de Arqueologia, 5 (1): 5-28.

Coelho M.D. \& CARdoso J.L. 2010/2011. O espólio malacológico do povoado calcolítico fortificado do Outeiro Redondo (Sesimbra). Contributo para o conhecimento das estratégias de recolecção de uma comunidade sedentária do $3 .^{\circ}$ milénio a.C. do litoral português. Estudos Arqueológicos de Oeiras,18: 235-286. 
CORRÊA, A.A.M. 1931. Les nouvelles fouilles à Muge. C.R. $X V^{\text {ème }}$ Congrès International d'Anthropologie et d'Archéologie préhistoriques. V'me Session de l'Institut International d'Anthropologie, Paris (1933): 357-372.

ClaAssen, C. 1998. Shells. Cambridge Manuals in Archaeology Series. Cambridge, New York, Melbourne, Cambridge University Press.

CosTA, F.A.P. 1865. Da existencia do homem em epochas remotas no valle do Tejo. Primeiro opusculo. Noticia sobre os esqueletos humanos descobertos no Cabeço da Arruda. Lisboa, Imprensa Nacional.

Clafssen, C. 2000. Animal remains, identification and analysis: molluscs. In: Ellis, L. (Ed.), Archaeological method and theory: an encyclopedia. New York and London, Garland publ.: 29-31.

Delgado, J.F.N. 1867. Da existencia do homem no nosso solo em tempos mui remotos provada pelo estudo das. Noticia ácerca das grutas da Cesareda. Lisboa, Typographia da Academia Real das Sciencias.

Detry, C. \& ArrudA, A.M. 2012. Acerca da influência ambiental e humana nos moluscos do Monte Molião (Lagos, Portugal). In: ALmeIDA, A.C.; Bettencourt, A.M.S.; Moura, D.; MonteIroRodrigues, S. \& Alves, M.I.C. (Eds.), Environmental Changes and Human interaction along the Western Atlantic edge/Mudanças ambientais e interação humana na fachada Atlântica ocidental, Coimbra, edição conjunta de APEQ, CITEM, CEGOT, CGUP,CCT: 171-184.

DEAN, R.M. \& CARVALHO, A.F. 2014. Faunal remains, adornments and bone tools. In: Carvalho, A.F. (Ed.), Bom Santo Cave (Lisbon) and the Middle Neolithic Societies of Southern Portugal. Faro, Universidade do Algarve: 195-205.

DinIZ, M. \& ARIAS, P. 2012. Human Settlement in the Sado Paleo-Estuary: some issues around Mesolithic Shell -Middens. Atas das IV Jornadas da APEQ. Universidade de Coimbra: 139-158.

FALKNER, G.; RIPKEN, T.E.J. \& FALKNER, M. 2002. Mollusques continentaux de France. Liste de référence annotée et bibliographie. Collection Patrimoines Naturels, 52. Paris, Publications Scientifiques du Muséum National d'Histoire Naturelle.

FERNANDEZ-LÓPEZ, S. 1999. Tafonomía y fosilización. In: Meléndez. B., Tratado de Paleontología, $3^{\mathrm{a}}$ Ed., vol. 1, cap. 3. Madrid, Consejo Superior de Investigaciones Científicas: 51-107.

FERrEIRA, O.V. 1956. Faune malacologique, crustacés et poissons. Muge (Moita do Sebastião). Actas IV Congreso Internacional de Ciencias Prehistóricas e Protohistóricas, Zaragoza: 339-346.

FERREIRA, O.V. \& Roche, J., 1980. Os elementos de adorno do Paleolítico Superior de Portugal. Arqueologia, 2: 7-11.

Garcia, V.B.; AgÜero, E.G.G. \& Rodríguez, C.F. 2010. La Arqueomalacología: una introducción al estudio de los restos de moluscos recuperados en yacimientos arqueológicos. Iberus, 28: 13-22.

GMELIN, J.F. (1791). Vermes. In: GMELIN J.F. (Ed.), Caroli a Linnaei Systema Naturae per Regna Tria Naturae. Editio Decima Tertia, Aucta Reformata. Tome 1, Pars 6 (Vermes). Lipsiae, Ed. G.E. Beer.
Gomes, R.V. \& Gomes, M.V. 2005. A djihād no Extremo Sudoeste Peninsular - O recém-identificado ribāt da Arifana (século XIII). Revista da Faculdade de Ciências Sociais e Humanas, 16: 141-159.

GonÇALVES, M.J. 2006. Alguns dados para a reconstituição dos hábitos alimentares da população de um Arrabalde da Silves Islâmica. Xelb, 6: 141-153.

Gonçalves, M.J.; Dias, R. \& Callapez, P.M. 2007. Espécies malacológicas presentes num contexto de lixeira de um Arrabalde da Silves Islâmica. Vipasca, Arqueologia e História, série 2, 2: 658-664.

GUTIÉRREZ ZuGASTI, I. 2008. Análisis tafonómico en arqueomalacología: el ejemplo de los concheros de la región cantábrica. Krei, 10: 53-74.

HOLYOAK, D.T. \& HolyOAK, G.A. 2010. A new species of Candidula (Gastropoda, Hygromiinae) from central Portugal. Iberus, 28(1): 67-72.

Holyoak, D.T. \& Holyoak, G.A. 2012. A taxonomic revision of Oestophora barbula (Rossmassler, 1838 ) and $O$. barbella (Servain, 1880), two Iberian endemic landsnail species (Gastropoda: Trissexodontidae). Iberus, 30 (1): 15-40.

Holyoak, D.T., Holyoak, G.A. \& Alba, J.S.T. 2012. A reassessment of the species of Truncatellina (Gastropoda: Vertiginidae) in the Iberian Peninsula and North-west Africa. Iberus, 30 (2): 7-33.

HolyoAK, D.T. \& Holyoak, G.A. 2015. A taxonomic review of Cecilioides (Gastropoda: Ferussaciidae) in continental Portugal. Iberus, 33: 27-43.

Kerney, M. \& CAMERON, R. 1979. A field guide to the land snails of Britain and North-West Europe. London, Ed. Collins.

LANDON, D.B. 2005. Zooarchaeology and Historical Archaeology: Progress and Prospects. Journal of Archaeological Method and Theory, 12 (1): 1-36.

LINK, D.H.F. 1807-1808. Beschreibung der NaturalienSammlung der Universität zu Rostock. Adlers Erben, Rostock

LINNAEUS, C. 1758. Caroli Linnai Systema natura per regna tria natura: secundum classes, ordines, genera, species, cum characteribus, differentiis, synonymis, locis, Ed. 10, reformata. Holmia, Impensis L. Salvii.

LINNAEUS, C. 1767. Systema naturae per regna tria naturae: Secundum classes, ordines, genera, species, cum characteribus, differentiis, synonymis, locis/Caroli a Linne. Editio decima tertia, ad editionem duodecimam reformatam Holmiensem. Vindobonae, Typis Ioannis Thomae.

LONGHURST, A. 1998. Ecological geography of the sea New York, Academic Press.

Lubell, D. 2004. Prehistoric edible land snails in the circum-Mediterranean: the archaeological evidence. In: Brugal, J.-P. \& Desse, J. (Eds.), Petits animaux et sociétés humaines. Du complément alimentaire aux ressources utilitaire, $\mathrm{XXIV}^{\mathrm{e}}$ Rencontres internationales d'Archéologie et d'Histoire d'Antibes. Antibes, Éditions APDCA: 77-98.

LYMAN, R.L. 1987. Zooarchaeology and taphonomy: a general consideration. Journal of Ethnobiology, 7 (1): 93-117.

Martins, S. 2013. Estudo arqueofaunístico do Castelo de Salir (Loulé) - Contribuição para o conhecimento 
da dieta alimentar islâmica. Tese de mestrado. Faro, Universidade do Algarve.

MARTYN, T. 1784-1787. The Universal Conchologist, exhibiting the figure of every known shells. 4 vols., London, edição do autor.

Macedo, M.C.C.; Macedo, M.I.C.; Borges, J.P. 1999. Conchas Marinhas de Portugal. Lisboa, Editorial Verbo.

MAtos, R.M.A. 1993a. Espèces de la famille Helicidae représentées dans la région agraire de l'Alentejo (Portugal). Vertigo, 3: 27-32.

MAtos, R.M.A. 1993b. Liste des gastéropodes terrestres, fluviatiles et d'eux saumâtres du Portugal. Tentative d'actualisation. Vertigo, 3: 33-45.

MAtos, R.M.A. 1994. Distribution of some helicid snails in Portugal. Brotéria Genética, 15: 29-36.

MAtos, R.M.A. 2004. Non-Marine Testaceous Gastropoda of Continental Portugal and Berlengas Islands. I. Catalogue and Bibliography. Arquivos do Museu Bocage, 4: 1-158.

Matos, R.M.A. 2014. Atlas dos caracóis terrestres e de águas salobras de Portugal Continental. Lisboa, Edição da autora.

MoORE, R.C. 1969. Treatise on Invertebrate Paleontology, part N, Mollusca 6 - Bivalvia. 3 vols. Boulder, Colorado, Univ. Kansas Press.

Moreno-Nuño, R., 1993. El Conjunto Malacológico del Yacimiento Portugués de Mertola (Campañas 1990/1991). Arqueologia Medieval, 2: 285-287.

Morton, B.; Britton, J. \& Martins, A.F. 1998. Ecologia costeira dos Açores. Ponta Delgada, Sociedade Afonso Chaves.

MÜLlER, O.F. 1773-1774. Vermium terrestrium et fluviatilium, seu animalium infusoriorum, helminthicorum, et testaecorum, non marinorum, succincta historia. 2 vols., Havniæ \& Lipsiæ, Heineck \& Faber.

NoBre, A. 1930. Moluscos terrestres, fluviais e das águas salobras de Portugal. Barcelos, Companhia Editora do Minho.

Nobre, A. 1931. Moluscos marinhos de Portugal, vol. 1. Porto, Imprensa Portuguesa.

NoBre, A. 1936. Moluscos marinhos de Portugal, vol. 2. Barcelos, Companhia Editora do Minho.

Nobre, A. 1940. Fauna malacológica de Portugal - I, Moluscos marinhos e das águas salobras. Barcelos, Imprensa Portuguesa, Companhia Editora do Minho.

Nobre, A. 1941. Fauna malacológica de Portugal - II, Moluscos terrestres e fluviais. Coimbra, Coimbra Editora.

NuÑo, R.M. 1993. El Conjunto Malacológico del Yacamiento Portugues de Mértola. Arqueologia Medieval, 2: 285-287.

OCAÑA, T.M.J. 2003. Growth, mortality and longevity in two populations of Siphonaria pectinata (Pulmonata) at Gibraltar. Journal of Molluscan Studies, 69(2): 162-164.
OLIVEIRA, F.P.E. 1892. Nouvelles fouilles faites dans les kjoekkenmoeddings de la vallée du Tage (mémoire posthume). Communicações da Comissão dos Trabalhos Geológicos, 2 (1): 57-81.

PAÇO, A. 1938. Novos concheiros do Vale do Tejo. Brotéria, 27 (1): 66-75.

Paredes, R.; Callapez, P.M.; DANIElsen, R.; Dinis, P.; CARvalho, M. \& SoAres, A.F. 2006. Paleoecologia da malacofauna salobra e biofácies da laguna holocénica de Leirosa (Figueira da Foz). VII Congresso Nacional de Geologia, Estremoz: 737-740.

PhILIPPI, R.A. 1844. Nachtrag zum zweiten Bande der Enumeratio Molluscorum Siciliae. Zeitschrift für Malakozoologie. 1: 100-112.

Poppe, G. \& Goto, Y. 1991. European Seashells, vol. IPolyplacophora, Caudofoveata, Solenogastra, Gastropoda. Wiesbaden, Verlag Christa Hemmen.

Poppe, G. \& Goto, Y. 1993. European Seashells, vol.II Scaphopoda, Bivalvia, Cephalopoda. Wiesbaden, Verlag Christa Hemmen.

REIS, J. 2006. Atlas dos bivalves de água doce de Portugal. Lisboa, Instituto da Conservação da Natureza.

RIBEIRO, C. 1884. Les kjoekkenmoeddings de la vallée du Tage. Congrès International d'Anthropologie et d'Archéologie Préhistoriques (Lisbonne, 1880). Compte-Rendu de la neuvième session. Lisbonne, Typographie de l'Académie Royale des Sciences: 279-289.

Roche, J. 1951. L'industrie préhistorique de Cabeço da Amoreira (Muge). Porto, Instituto para a Alta Cultura, Centro de Estudos de Etnologia Peninsular.

Roche, J. 1953a. Les fouilles des amas coquilliers de Muge. Leur importance pour la chronologie du Mésolithique. Boletim da Sociedade Geológica de Portugal, $10: 145-150$

Roche, J. 1953b. Note sur les méthodes de fouille utilisées lors des explorations des amas coquilliers de Muge. Naturalia, série 2, 1 (1): 29-33.

Roche, J. 1960. Le gisement Mésolithique de Moita do Sebastião. Muge, Portugal. I-Archéologie. Lisboa, Instituto de Alta Cultura.

Roche, J. 1972. Les amas coquilliers (concheiros) mésolithiques de Muge (Portugal). In: H. Sschwabedissen, Die Anfänge des Neolithikums vom Orient bis Nordeuropa, Fundamenta, 7 : 72-107.

RodrígueZ, R.G. \& SÁNCHEZ, J.M. 1997. Moluscos bivalvos de Canárias. Las Palmas, Cabildo Insular de Gran Canaria.

RolÁn, E. 1984. Moluscos de la Ria de Vigo. I- Gasteropodos. Santiago de Compostela, Ed. Velograf S.A

Rolán, E.; Schmitt, J.O. \& AlvareZ, E.R. 1989. Moluscos de la Ria de Vigo. II - Poliplacoforos, Bivalvos, Escafópodos, Cefalopodos. Thalassas, Anexo 2, 276 p.

SALDANHA, L. 1997. Fauna Submarina Atlântica, $3^{\text {a }}$ edição. Lisboa, Publicações Europa-América.

Silva, C.M. 1997. Malacofauna da Tróia Romana. In: AAVV, Portugal Romano. A Exploração dos Recursos Naturais. Lisboa, Museu Nacional de Arqueologia: 65. 
Silva, C.M. 2000. Les Mollusques d'Abul A. In: Mayet, F. \& Silva, C.T. (Eds.), Le établissement Phénicien d'Abul (Portugal), Appendice V. Paris, Diffusion E. de Boucard: 293-303.

Silva, C.T. \& CABritA, M.G. 1966. A utilização dos moluscos durante o Eneolítico português. Revista de Guimarães, 76 (3-4): 307-338.

Silva, C.T. \& SoARES, J. 1993. Na transição PlistocénicoHolocénico. Marisqueio na Pedra do Patacho. AlMadan, série 2, 2: 21-29.

SiLVA, C.T. 1996. Malacofauna e Arqueologia. Al-Madan, série 2, 5: 89-95.

Silva, C.T. \& SoAres, J. 1997. Economias costeiras na Préhistória do Sudoeste Português. O concheiro de Montes de Baixo. Setúbal Arqueológica, 11-12: 69-108.

Silva, C.T. \& SOARES, J. 1998. Os recursos marinhos nas estratégias de subsistência da Pré-História do Sul de Portugal. Al-Madan, série 2, 7: 71-82.

SousA, A.C. 1998. O Neolítico Final e o Calcolítico na área da Ribeira de Cheleiros. Trabalhos de Arqueologia, 11: 1-275.

Spalding, M.D.; FoX, H.E.; Allen, G.R.; DAVIDSON, N.; FERdaña, Z.A.; Finlayson, M.; HALPERN, B.S.; Jorge, M.A.; LOMBANA, A.; LOURIE, S.A.; MaRTIN, K.D.; MANUS, E.M.C.; MOLNAR, J.; RECCHIA C.A. \& RoberTSON, J. 2007. Marine Ecoregions of the World: A Bioregionalization of Coastal and Shelf Areas. BioScience, 75(7): 573-583.

TÁtÁ, F.; CAscalheira, J.; Marreiros, J.; Marreiros, T.; Pereira, T. \& Bicho, N. 2013. Shell bead production in the Upper Paleolithic of Vale Boi (SW Portugal): an experimental perspective. Journal of Archaeological Science, 42: 29-41.

TEBBLE, N. 1976. British bivalve seashells. $2^{\text {nd }}$ edition. Edinburgh, Royal Scottish Museum.

VALENTE, M.J. 2010a. O Barranco das Quebradas (Vila do Bispo) no contexto dos concheiros mesolíticos do Sudoeste português. Xelb, 10: 16-38.

VAlente, M.J. 2010b. Pequeno guia para identificação dos moluscos marinhos em contextos arqueológicos. Faro, Departamento de História, Arqueologia e Património, Universidade do Algarve.
VALENTE, M.J. \& MARTINS, S. 2015. Os moluscos marinhos como recurso alimentar no Garb al-Andalus: os sítios de Cacela Velha (Vila Real de Santo António) e Castelo de Salir (Loulé). In: Zugasti, I.G.; SolanA, D.C. \& Morales, M.R.G. (Eds.), La investigación arqueomalacológica en la Península Ibérica: nuevas aportaciones. Santander, Nadir Ediciones: 199-212.

VANHAEREN, M. \& D'ERRICO, F. 2002. The body ornaments associated with the burial. In: ZILHÃO, J. \& Trinkaus, E. (Eds.), Portrait of the Artist as a Child. The Gravettian Human Skeleton from the Abrigo do Lagar Velho and its archaeological context. Lisboa, IPA: 154-186.

VANTREPOTte, V. \& MÉLin, F. 2009. Temporal variability of 10-year global SeaWiFS time series of phytoplankton chlorophyll a concentration. ICES Journal of Marine Science, 66: 1547-1566.

VermeIJ, G.J. 2001. Distribution, history, and taxonomy of the Thais clade (Gastropoda: Muricidae) in the Neogene of tropical America. Journal of Paleontology, 75 (3): 697-705.

WENZ, W. 1938-44. Gastropoda, Teil 1: Allgemeiner teil und Prosobranchia. Handbuch Paläozoologie, 6: 11639.

ZByszewsKi, G.; FERREIRA, O.V.; LEITÃO, M.; NORTH, C.T. \& NorTON, J. 1981. Nouvelles données sur le Néolithique ancien de la station à céramique cardiale de Sagres, Algarve. Comunicações dos Serviços Geológicos de Portugal, 67: 301-311.

ZILCH, A. 1960. Gastropoda, vol. 2: Euthyneura. Berlin, Gebrüder Borntraeger.

ZILHÃo, J. 1992. Gruta do Caldeirão: O Neolítico Antigo. Trabalhos de Arqueologia, 6: 1-326.

ZILHÃo, J. 1997. O Paleolitico Superior da Estremadura Portuguesa. vol. 2, Lisboa, Edições Colibri.

Zilhão, J.; ANGEluCCI, D.; BADAL-GARCÍA, E.; D’ERrico, F.; DANiel, F.; DAYet, L.; DoukA, K.; HighaM, T.; Martínez-SÁnCHEZ, M.; MONTESBernárdez, R.; Murcia-Mascarós, S.; PÉrezSiRVENT, C.; RoldÁN-GARCÍA, C.; VANHAEREN, M.; Villaverde, V.; WoOd, R. \& Zapata, J. 2010. Symbolic use of marine shells and mineral pigments by Iberian Neandertals. Proceedings of the National Academy of Sciences, 107: 1023-1028. 